\title{
Quels impacts des changements climatiques sur les eaux de surface en France à l'horizon 2070 ?
}

\author{
Mathilde CHAUVEAU ${ }^{1,2}$, Sébastien CHAZOT ${ }^{1}$, Charles PERRIN ${ }^{2}$, Pierre-Yves BOURGIN ${ }^{2,7}$, \\ Eric SAUQUET ${ }^{3}$, Jean-Philippe VIDAL ${ }^{3}$, Nathalie ROUCHY ${ }^{4}$, Eric MARTIN ${ }^{4}$, \\ Julian DAVID ${ }^{1}$, Thomas NOROTTE ${ }^{1}$, Pascal MAUGIS ${ }^{5,8}$, Xavier DE LACAZE ${ }^{6}$
}

\author{
BRLi, Nîmes. \\ 2. Irstea, UR HBAN, Antony. \\ 3. Irstea, UR HHLY, Lyon. \\ 4. Météo-France, Toulouse. \\ 5. ONEMA, Vincennes. \\ 6. DEB/DGALN, MEDDE, La Défense. \\ 7. Maintenant à Tractebel Engineering SA, Gennevilliers. \\ 8. Maintenant au LSCE (CEA-CNRS-UVSQ), IPSL, Gif-sur-Yvette.
}

\begin{abstract}
RÉSUMÉ. - Dans le cadre du projet Explore 2070, une évaluation des impacts possibles des changements climatiques sur les eaux de surface à l'horizon 2046-2065 par rapport à un état de référence 1961-1990 a été réalisée en France métropolitaine et sur les départements d'Outre-Mer sur la base du scénario d'émission de gaz à effet de serre A1B, de sept modèles climatiques et deux modèles hydrologiques (Isba-Modcou et GR4J). Sur la métropole, les résultats obtenus indiquent : (1) une augmentation possible des températures moyennes annuelles de l'air de l'ordre de $+1,4^{\circ} \mathrm{C}$ à $+3^{\circ} \mathrm{C}$; (2) une évolution incertaine des précipitations, la plupart des modèles s'accordant cependant sur une tendance à la baisse des précipitations en été sur l'ensemble de la métropole ; (3) une diminution significative globale des débits moyens annuels à l'échelle du territoire, de l'ordre de $10 \%$ à $40 \%$, particulièrement prononcée sur les districts SeineNormandie et Adour-Garonne ; (4) pour une grande majorité des cours d'eau, une baisse prononcée des débits d'étiage ; (5) des évolutions plus hétérogènes et globalement moins importantes sur les crues. Une attention particulière a été donnée à l'évaluation des incertitudes associées à ces résultats. Elles permettent de mieux évaluer le niveau de signification des évolutions projetées. Les tendances calculées pour les départements d'Outre-Mer peuvent être considérées comme non significatives compte tenu du niveau d'incertitude élevé associé à la chaîne de modélisation hydro-climatique. Les résultats obtenus invitent à définir dès à présent des stratégies d'adaptation basées notamment sur une meilleure gestion de la ressource.
\end{abstract}

Mots-clés : changement climatique, impacts hydrologiques, France, incertitudes, multi-modèle

\section{What will be the impacts of climate change on surface hydrology in France by 2070?}

\begin{abstract}
Within the Explore 2070 project, an evaluation of the possible impacts of climate change on surface water between the 1961-1990 reference period and the 2046-2065 period was carried out in continental France and in overseas departments on the basis of the A1B greenhouse gas emission scenario, seven general circulation models and two hydrological models (Isba-Modcou and GR4J). In continental France, results indicate: (1) a possible increase in air temperature between $+1.4^{\circ} \mathrm{C}$ and $+3^{\circ} \mathrm{C}$; (2) an uncertain evolution of precipitation, most models however agreeing on a decreasing trend in summer precipitation; (3) a significant decrease (10\% to $40 \%$ ) of mean annual flows at the country scale, especially pronounced in the Seine-Normandie and Adour-Garonne districts; (4) a strong decrease in summer low flows in most basins; (5) more heterogeneous and less significant evolutions for floods. A special care was given to the quantification of the uncertainties linked to these results. They provide an indication of the significance of projected changes. The evolutions calculated in the overseas zones can be considered non-significant given the level of uncertainty linked to the hydro-climatic modelling chain. These results urge to implement adaptation strategies based on a better management of water resources, among others.
\end{abstract}

Key-words: climate change, hydrological impacts, France, uncertainties, multimodel

\section{INTRODUCTION}

Les questions relatives à la disponibilité et à la gestion de l'eau concentreront l'essentiel des mesures d'adaptation qui seront prises dans les décennies à venir pour faire face aux conséquences des changements climatiques. Il est dès lors primordial pour les décideurs et gestionnaires de quantifier le devenir de la ressource en eau et de sa variabilité temporelle et spatiale.

C'est dans ce contexte que le projet Explore 2070, initié par le Ministère de l'Écologie, du développement durable et de l'énergie, a eu pour objectif d'évaluer les impacts possibles des changements climatiques et socioéconomiques futurs sur les grandes masses d'eau en France 
métropolitaine et dans les départements d'Outre-Mer, à l'horizon 2050-2070.

La quantification des impacts des changements climatiques sur les eaux de surface a déjà fait l'objet de nombreuses études, en France et à l'étranger, à des échelles spatiales et temporelles variées et avec des objectifs de quantification différents sur la ressource, les étiages, les crues, etc. Les travaux de Boé [2007] et de Boé et al. [2009] mettaient en œuvre une chaîne de modélisation uniforme sur l'ensemble du territoire français. Les projections hydrologiques obtenues avec le modèle hydrologique IsbaModcou [Habets et al., 2008] indiquent une diminution des débits moyens en été et en automne, ainsi qu'une diminution de l'humidité du sol et de la couverture neigeuse, spécialement à basse et moyenne altitude. Les étiages sévères voient leur fréquence s'accroître et les évolutions sur les crues sont incertaines. Le projet Climsec [Soubeyroux et al., 2011 ; Najac et al., 2010 ; Vidal et al., 2012] s'est attaché à évaluer les impacts possibles du changement climatique sur l'eau du sol et les phénomènes de sècheresse à l'échelle nationale, au cours du $\mathrm{XXI}^{\mathrm{e}}$ siècle. Une approche multimodèle permettant une analyse des sources d'incertitudes a été mise en œuvre. L'étude conclut à des aggravations majeures des sécheresses agricoles en termes d'extension spatiale ou d'intensité à l'horizon 2050. A l'horizon 2080, des sécheresses météorologiques plus fortes pourraient ainsi apparaître, notamment en été, et les sécheresses du sol pourraient devenir extrêmes sur la majeure partie du territoire. Notons que de nombreuses études, comme le projet RExHySS sur la Seine et la Somme [Ducharne et al., 2011], ont également été menées à l'échelle de districts hydrographiques ou grands bassins français [voir par exemple la synthèse réalisée par Perrin et al., 2010].

Dans le cadre du projet Explore 2070, une chaîne de modélisation multi-modèles a été mise en place pour permettre le traitement uniforme de tout le territoire métropolitain, avec une attention particulière portée à la quantification des incertitudes associées aux modèles climatiques et hydrologiques. Les impacts sur le régime et les aléas hydrologiques (débits mensuels et annuels, caractéristiques de crue et d'étiage) sont étudiés sur l'ensemble du territoire, à l'exutoire de plus d'un millier de bassins versants. Les résultats produits aideront les décideurs à mieux concevoir les stratégies d'adaptation nécessaires pour faire face aux évolutions projetées de la ressource.

Nous détaillerons dans ce qui suit la démarche générale de modélisation proposée, les résultats obtenus, ainsi que la façon dont les incertitudes ont été quantifiées. Ce dernier point est indispensable pour aider à la prise de décision dans un avenir incertain. Nous présentons ici essentiellement les résultats obtenus sur les débits en métropole [voir Chazot et al., 2012b pour plus de détails]. Notons que d'autres travaux ont été réalisés dans le cadre du projet Explore 2070 sur l'évolution des débits dans les départements d'OutreMer [Chazot et al., 2012c] ou des températures de l'eau en métropole [Chazot et al., 2012a].

\section{DONNEES ET METHODES}

Une chaîne de modélisation a été mise en œuvre afin de produire des simulations de débits journaliers en temps présent (1962-1991, période de référence) et en temps futur (2046-2065) pour 1522 points de calcul sur la métropole. La comparaison de ces simulations doit permettre d'estimer les impacts possibles du changement climatique sur l'hydrologie de surface.

\section{II.1. Données hydroclimatiques}

L'ensemble des 1522 points de calcul (Figure 1) a été sélectionné afin de constituer un échantillon le plus représentatif possible de la variabilité des conditions hydroclimatiques et des enjeux à l'échelle nationale, tout en garantissant une couverture relativement homogène du territoire, en fonction des données disponibles et des contraintes de modélisation (application possible d'un ou deux modèles hydrologiques).

Des observations hydrométéorologiques fournissent un cadre d'analyse de référence pour la période actuelle : la base de données de la Banque Hydro (www.hydro.eaufrance.fr) pour les chroniques hydrologiques, et la base de données SAFRAN $(8 \times 8 \mathrm{~km})$ de Météo-France [Quintana-Seguí et al., 2008 ; Vidal et al., 2010] pour les données météorologiques, ont été mobilisées. Elles sont utilisées sur la période de

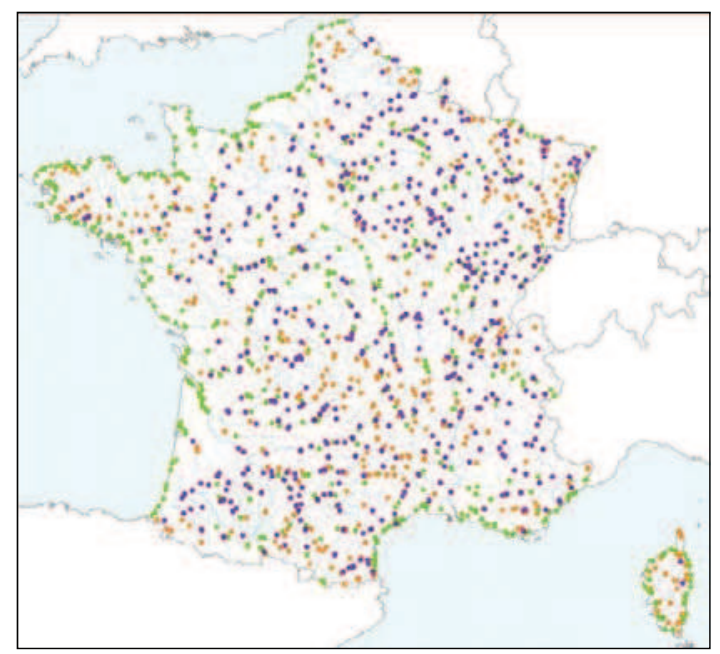

Points de calcul Explore 2070

- Points doubles GR4J + IsbaModcou (543 points)

- Points GR4J seul (359 points)

- Points Isba-Modcou seul (620)

Figure 1 : Localisation des 1522 points de calcul hydrologiques retenus dans Explore 2070, distingués en fonction des modèles hydrologiques appliqués. 
référence 1962-1991, dans la mesure de la disponibilité des données hydrométriques.

Pour la production de projections hydrologiques, des simulations climatiques issues de sept modèles de circulation générale (GCMs) forcés par le scénario d'émission de gaz à effet de serre $\mathrm{A} 1 \mathrm{~B}$, et désagrégées par la méthode de descente d'échelle statistique par type de temps DSCLIM [Boé et al., 2007], ont été utilisées. Ces projections climatiques sont issues des simulations réalisées dans le cadre du quatrième rapport du Groupe d'Experts Intergouvernemental sur l'Évolution du Climat (IPCC AR4) et des travaux du CERFACS [Pagé et al., 2008]. Sept GCMs ont été retenus (CCMA-CGCM3, ECHAM5/MPI, ARPEGE V3+, GFDL-CM2.0, GFDL-CM2.1, GISS MODEL ER, MRICGCM2.3.2) car ils représentent la variabilité des simulations climatiques de la vingtaine de modèles climatiques utilisés pour le rapport. Ces résultats fournis par MétéoFrance sont des chroniques de variables météorologiques de surface, y compris l'évapotranspiration potentielle selon la formulation de Penman-Monteith, au pas de temps journalier et sur un maillage régulier de $8 \times 8 \mathrm{~km}$. L'approche multimodèles doit permettre de rendre compte d'une partie des incertitudes liées aux GCMs. Les incertitudes liées au scenario d'émission de gaz à effet de serre et à la méthode de descente d'échelle ne sont en revanche pas quantifiées dans cette étude, bien qu'elles puissent fortement influer sur les résultats [Quintana-Seguí et al., 2010].

\section{II.2. Modélisation hydrologique}

Ces simulations climatiques sont ensuite utilisées pour alimenter deux modèles hydrologiques de conception très différentes, GR4J [Perrin et al., 2003] et Isba-Modcou [Habets et al., 2008].

Le modèle GR4J est un modèle pluie-débit à réservoirs et à quatre paramètres, fonctionnant à l'échelle globale $\mathrm{du}$ bassin versant. Il a déjà été testé sur de très nombreux bassins versants français [par ex. Le Moine et al., 2007]. Le modèle GR4J a été calé pour chaque station d'étude où l'on disposait de suffisamment de données sur la période de référence, grâce aux observations hydrométéorologiques. On fait l'hypothèse dans ces calages que les données de débit utilisées sont représentatives d'un fonctionnement naturel, bien que ce ne soit pas toujours le cas du fait de l'influence des prélèvements et/ou des ouvrages hydrauliques. Le paramétrage résultant est utilisé pour la simulation hydrologique sous changement climatique.

Le modèle Isba-Modcou est un modèle distribué à base physique composé d'un schéma de surface, Isba (Interaction Surface-Biosphere-Atmosphere) et d'un modèle hydrogéologique, Modcou, qui simule les transferts d'eau faisant intervenir la rivière et la nappe aquifère [Soubeyroux et al., 2008, 2010]. La chaîne SafranIsba-Modcou (SIM) a été développée dans le cadre d'une coopération entre le CNRM-GAME de Météo-France et le Centre de Géosciences de Mines ParisTech. Contrairement à GR4J, les paramètres du modèle Isba-Modcou n'ont pas fait l'objet d'un calage pour chaque station hydrométrique. Les simulations Isba-Modcou utilisées dans le cadre du projet Explore 2070 sont celles produites par Boé et al. [2009]. Les points de simulation Isba-Modcou ayant été définis antérieurement au projet, seuls 543 points de simulations sont communs avec ceux choisis pour GR4J. Nous présentons ici les résultats obtenus au droit de ces 543 points de calcul uniquement, afin de pouvoir appréhender l'incertitude liée aux modèles hydrologiques.
En amont de la simulation sous changement climatique, une étape d'évaluation des deux modèles en conditions actuelles a été menée, afin d'estimer la fiabilité des modèles et de donner ainsi une indication de leur performance possible en conditions futures.

La démarche de modélisation hydrologique adoptée repose sur de fortes hypothèses, concernant notamment :

- la stationnarité du bassin : il est supposé que le fonctionnement des bassins versants et l'état de couverture du sol seront inchangés, de sorte que le paramétrage des modèles restera valable en conditions futures. Or l'évolution climatique peut avoir un impact sur la couverture végétale (naturelle ou agricole) et l'évapotranspiration et ainsi modifier les composantes du bilan en eau ;

- le biais des modèles : il est supposé que le biais des modèles hydrologiques en temps futur est du même ordre de grandeur que le biais en conditions actuelles;

- la capacité d'extrapolation des modèles : on suppose que le modèle peut s'adapter à des conditions pas ou peu rencontrées sur la période de référence choisie pour estimer les paramètres [Coron et al., 2012].

\section{II.3. Descripteurs hydrologiques et analyse des changements possibles}

Afin de caractériser les évolutions hydrologiques (régimes, étiages, crues) entre la période de référence et la période future, un ensemble de descripteurs, notamment les débits moyens annuels et mensuels, le débit minimum mensuel quinquennal sec (QMNA5) et le débit journalier maximal de période de retour 10 ans (QJXA10), a été calculé à partir des simulations de débits journaliers.

Pour chaque descripteur, une valeur de référence est calculée à partir des chroniques hydrométriques d'observation. Pour chaque combinaison de modèles climatique et hydrologique, l'écart entre simulation présente et simulation future donne le changement hydrologique relatif estimé, lequel est à rapporter à la valeur de référence. Cette méthode permet à la fois de comparer plus facilement entre elles les évolutions obtenues sur les différents bassins, et de s'affranchir partiellement du biais lié à la modélisation en temps présent. Elle fait l'hypothèse de constance du biais des modèles entre présent et futur. Elle rend cependant l'estimation dépendante de la qualité de la valeur de référence utilisée pour normer la différence entre présent et futur.

Pour chacun de 1522 points de calcul, une fiche synthétisant les résultats obtenus en termes d'évolutions climatiques et hydrologiques, caractérisées par une vingtaine d'indicateurs statistiques, a été produite (Figure 2).

\section{RESULTATS}

\section{III.1. Évaluation des modèles hydrologiques}

La capacité des modèles hydrologiques à reproduire les débits actuels, et en particulier les situations de hautes et basses eaux, a été évaluée à l'aide des critères quadratiques de Nash-Sutcliffe [1970] sur les débits (donnant plus d'importance aux crues) et sur les logarithmes des débits (donnant plus d'importance aux périodes de basses eaux). D'autres critères de type événementiel plus spécifiques aux crues ou aux étiages ont également été calculés mais ne sont pas détaillés ici. Ils sont illustrés à la Figure 2.

Le modèle Isba-Modcou ne se prêtant pas à une analyse en calage-contrôle [Klemeš, 1986], les simulations d'Isba-Modcou 


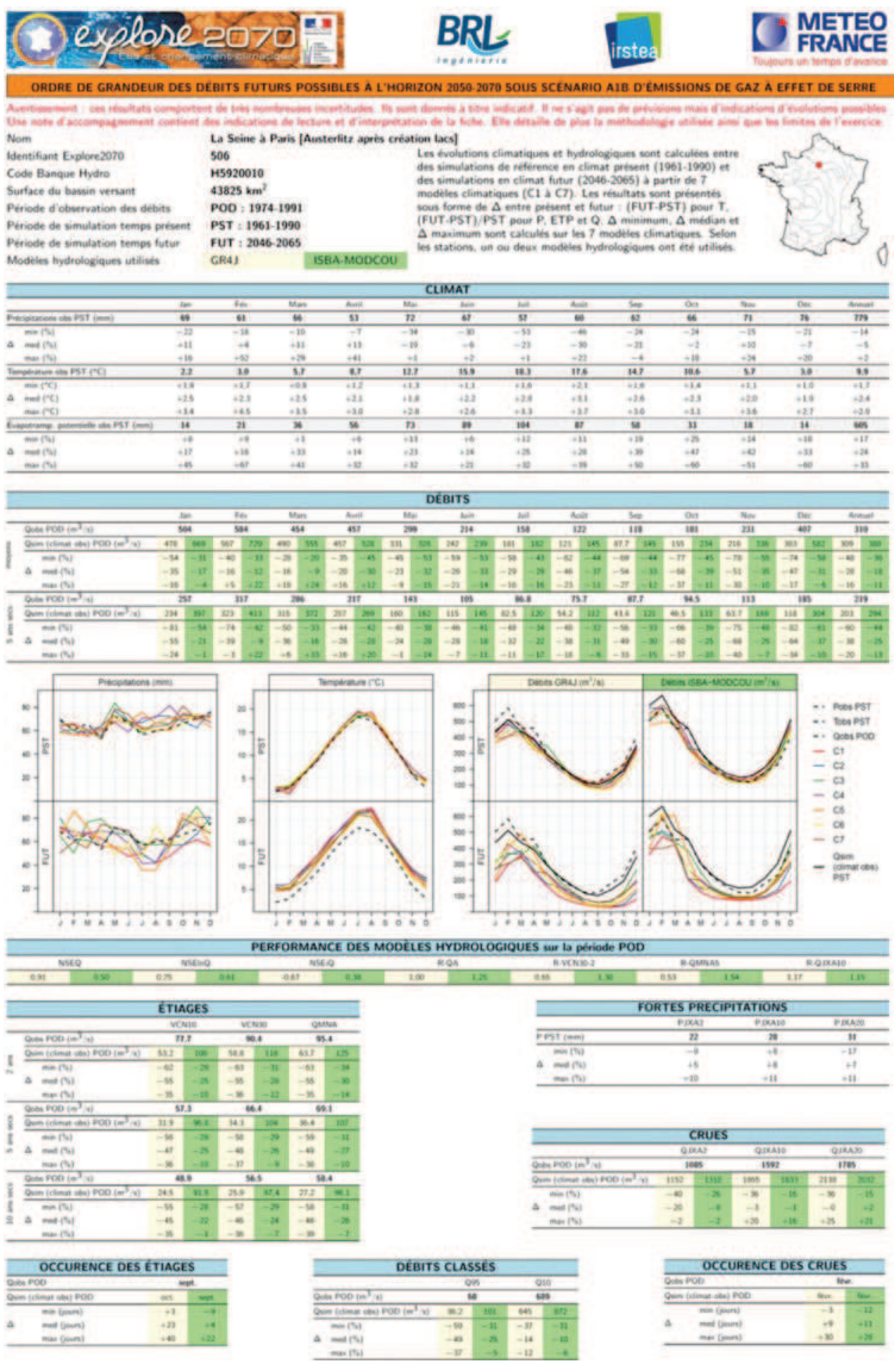

Figure 2 : Exemple de fiche de synthèse des changements climatiques et hydrologiques, produite pour chaque bassin versant d'étude (ici la Seine à Paris-Austerlitz). De haut en bas: caractéristiques générales du bassin; évolutions climatiques mensuelles et annuelles; évolutions des débits annuels et mensuels ; graphes d'évolution des régimes climatiques et hydrologiques ; performance des modèles en temps présent ; évolution d'indicateurs d'étiage et de crue. Les sept modèles climatiques C1 à C7 sont ceux définis dans la partie II.1 et les deux modèles hydrologiques sont GR4J et Isba-Modcou.

ont été comparées à celles obtenues en calage par GR4J sur l'ensemble de la période de référence pour les 543 points où les deux simulations hydrologiques étaient disponibles. En parallèle, des tests supplémentaires en calage-contrôle sur des périodes indépendantes ont été réalisés pour évaluer la fiabilité du modèle GR4J (résultats légèrement moins bons, non détaillés ici). Les résultats (résumés dans le Tableau 1) montrent que le modèle GR4J donne des résultats globa- lement satisfaisants en temps présent, avec cependant des difficultés de modélisation sur la Corse, le pourtour méditerranéen, la Seine moyenne et aval et le Nord de la France. Comparativement, les performances du modèle Isba-Modcou sont moins bonnes en temps présent, avec plus de difficultés dans la simulation des basses eaux que des hautes eaux. Ceci s'explique en partie par le fait qu'Isba-Modcou n'a fait l'objet que d'un calage global des paramètres pour équilibrer 
Tableau 1: Critères de Nash-Sutcliffe (NS) calculés sur les débits (Q) et sur les logarithmes des débits (lnQ) pour les modèles GR4J et Isba-Modcou, sur les 543 sous-bassins versants simulés par les deux modèles. Quantiles $10 \%, 50 \%$ et $90 \%$ des 543 valeurs du critère.

\begin{tabular}{|l|c|c|c|c|c|c|}
\hline & \multicolumn{3}{|c|}{ NS(Q) } & \multicolumn{3}{c|}{ NS(lnQ) } \\
\hline & GR4J & Isba-Modcou & GR4J en non jaugé & GR4J & Isba-Modcou & GR4J en non jaugé \\
\hline Quantile $10 \%$ & 0.67 & -0.70 & 0.37 & 0.62 & -3.7 & 0.18 \\
\hline Médiane & 0.83 & 0.54 & 0.76 & 0.80 & 0.13 & 0.73 \\
\hline Quantile 90\% & 0.90 & 0.74 & 0.87 & 0.89 & 0.69 & 0.86 \\
\hline
\end{tabular}

les bilans à l'échelle des grands bassins, et non à chaque station comme GR4J : les différences de performance sont légèrement moins importantes quand ce dernier est appliqué sur les mêmes stations considérées non jaugées, avec estimation des paramètres par transposition à partir des bassins versants voisins jaugés [Oudin et al., 2008]. Cette évaluation permet d'estimer la fiabilité des modèles et une part de l'incertitude liée à la modélisation hydrologique. Il s'agit cependant d'une évaluation partielle, qui ne permet pas de tester les hypothèses de fiabilité de ces modèles sous changement climatique et leur capacité d'extrapolation.

\section{III.2. Projections climatiques}

Tous les modèles étudiés simulent une hausse des températures assez uniforme sur le territoire, comprise entre $1,4^{\circ} \mathrm{C}$ et $3^{\circ} \mathrm{C}$ en moyenne annuelle (Figure 3 ). En revanche, une grande disparité entre modèles apparait sur les évolutions des précipitations saisonnières. La plupart des modèles s'accordent néanmoins sur une tendance à la baisse des précipitations en été sur l'ensemble du territoire, de l'ordre de $-16 \%$ à $-23 \%$. Aucune évolution significative générale ne se dessine en hiver et au printemps à l'échelle du territoire. L'estimation de l'évolution des précipitations et donc du cycle hydrologique est ainsi soumise à de fortes incertitudes.

\section{III.3. Évolution de la ressource en eau annuelle}

Il est important de considérer simultanément les changements hydrologiques simulés et l'incertitude associée à ces résultats. Afin de prendre en compte ces éléments, la Figure 4 représente, pour chaque point d'étude où les deux modèles hydrologiques ont été utilisés, le changement moyen calculé et l'écart-type relatif des quatorze simulations. On suppose que la concordance des simulations entre modèles traduit une plus grande fiabilité des résultats. Notons qu'il demeure une assez forte dispersion des résultats même pour les cas où les simulations semblent les plus convergentes. En complément, la Figure 5 rend compte des résultats des 14 simulations pour quatre grands bassins versants.

Les sorties des simulations s'accordent sur une baisse globale du débit moyen annuel sur toute la métropole, à l'exception des affluents rive droite du Bas-Rhône sur lesquels les différentes projections sont en désaccord. Pour une majorité de points, le débit moyen annuel pourrait baisser de l'ordre de 10 à $40 \%$. Les modèles projettent une baisse particulièrement marquée de débit moyen annuel pour les cours d'eau des contreforts pyrénéens, et dans une moindre mesure, de la majorité du district hydrographique SeineNormandie, avec des changements simulés compris entre -10 et $-60 \%$. Ces territoires semblent subir l'effet combiné d'une hausse de l'évapotranspiration résultant d'une hausse de la température et d'une baisse relative des précipitations (Figure 3). La plupart des simulations projettent une baisse des débits moyens mensuels sur la majeure partie de l'année pour les bassins du Rhône, de la Seine, la Loire et la Garonne, à l'exception des débits des mois de février à avril, pour lesquels les résultats sont plus contrastés (Figure 5). La baisse pourrait être particulièrement marquée sur le bassin de la Garonne.

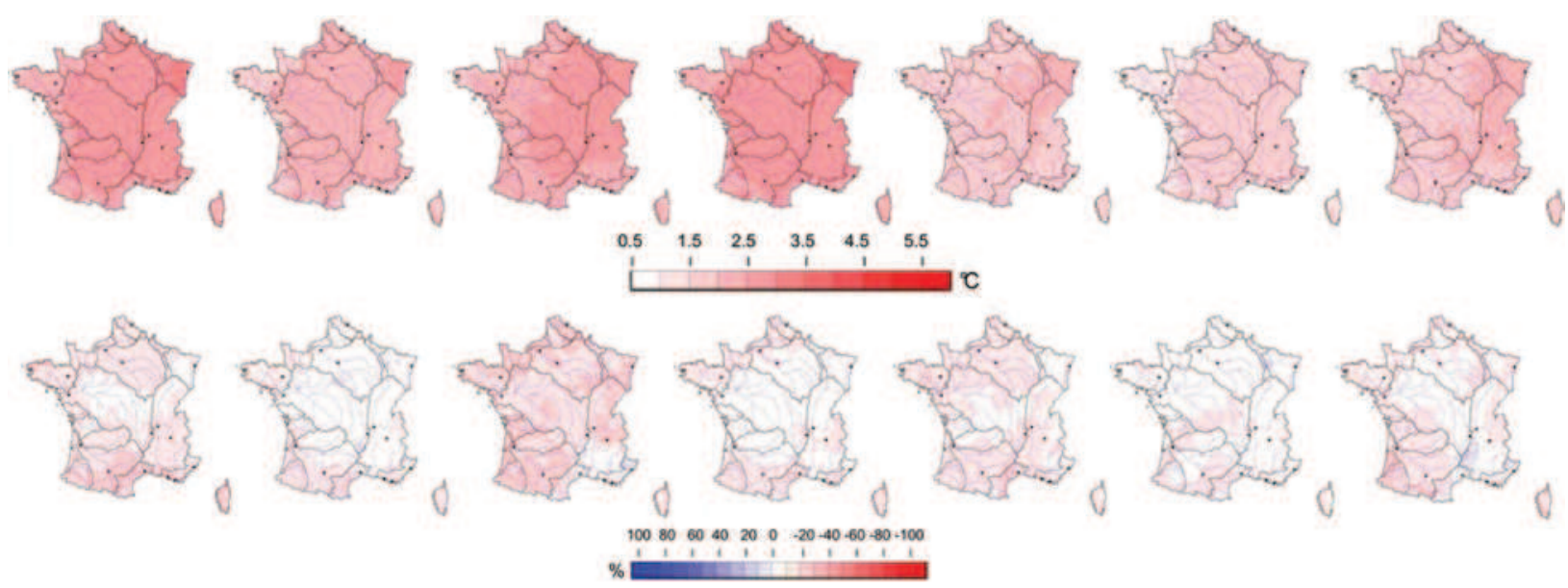

Figure 3 : Évolutions de températures annuelles (en haut, en ${ }^{\circ} \mathrm{C}$ ) et des cumuls de précipitations annuels (en bas, en \%) projetées par les sept GCMs (de gauche à droite) entre 1961-1991 et 2045-2067. 


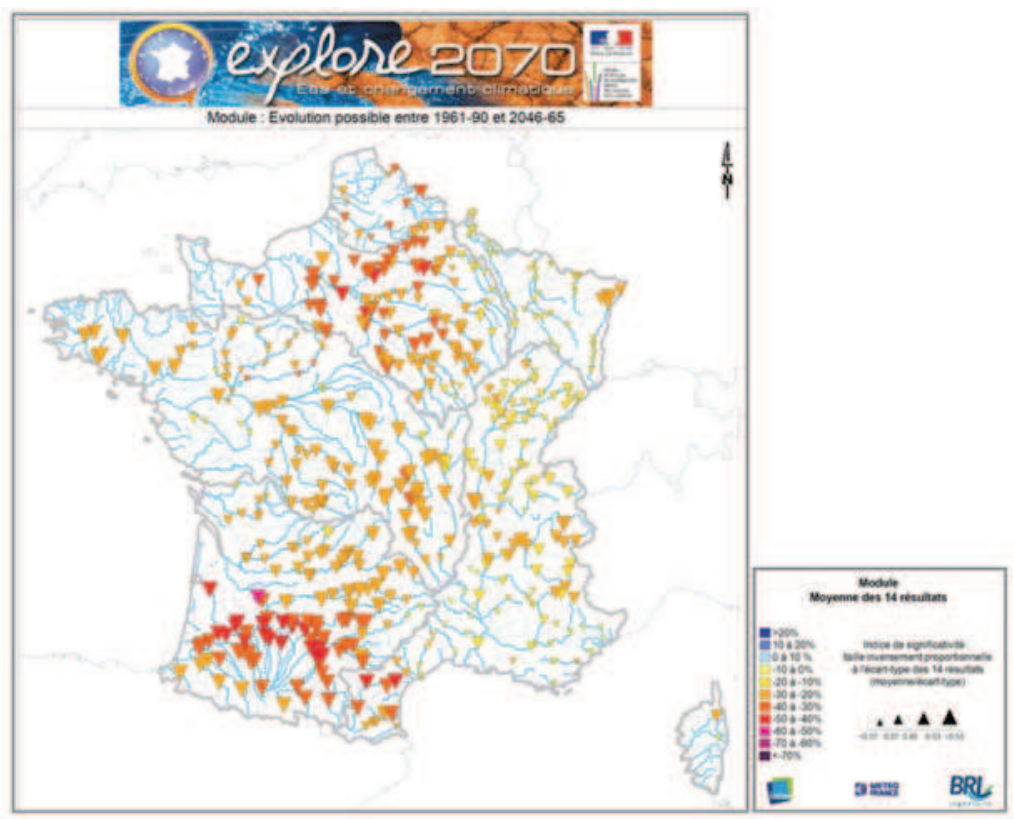

Figure 4 : Evolutions relatives possibles (en \%) du débit moyen annuel (module) entre 1961-90 et 2046-65: Résultats moyens établis sur les 14 simulations (2 modèles hydrologiques $\times 7$ GCMs). La couleur des points est fonction de l'intensité du changement et la taille des points est liée à la convergence des 14 simulations.
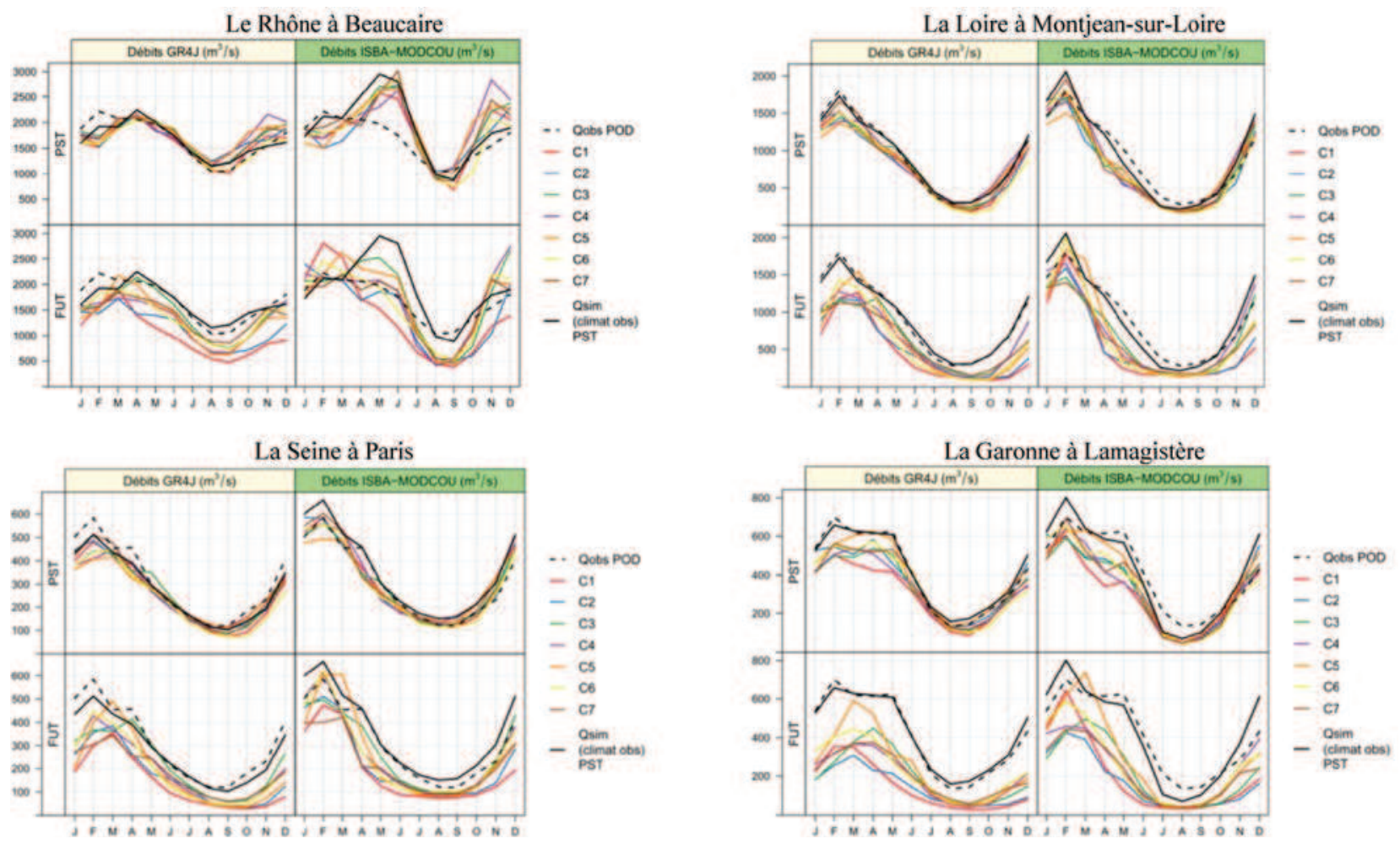

Figure 5 : Débits moyens mensuels simulés par les sept modèles climatiques et les deux modèles hydrologiques GR4J (colonnes de gauche) et Isba-Modcou (colonnes de droite) en temps présent (PST, premières lignes) et en temps futur (FUT, secondes lignes) pour quatre stations hydrométriques. "Cl” à "C7" renvoient aux simulations issues des 7 GCMs. Qobs POD sont les débits observés mesurés sur la période d'observation des débits ; Qsim PST sont les débits simulés par les modèles hydrologiques forcés par les données météorologiques SAFRAN sur la période de référence.

\section{III.4. Évolution des étiages}

Les projections s'accordent sur une aggravation générale des étiages pour la majorité des bassins de la métropole (Figure 6). On note une plus grande dispersion des résultats, comparativement aux changements sur les débits annuels, avec cependant des baisses plus sévères des débits d'étiages sur la majeure partie du territoire, en particulier pour les projections issues du modèle GR4J. Dans les bassins de montagne, les projections s'accordent globalement sur des 


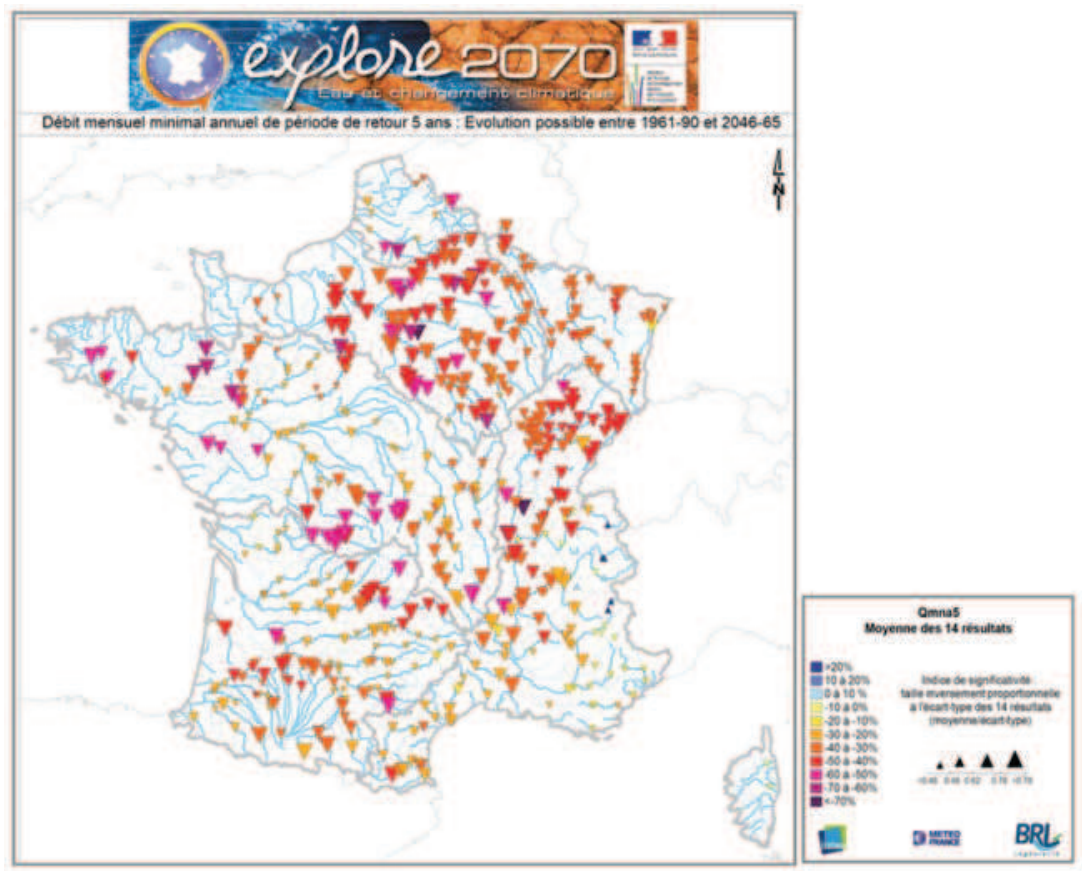

Figure 6 : Évolutions relatives possibles (en \%) du QMNA5 entre 1961-90 et 2046-65: Résultats moyens établis sur les 14 simulations (2 modèles hydrologiques $\times 7$ GCMs).

étiages estivaux plus sévères, mais les projections divergent concernant l'évolution des étiages hivernaux (non montré ici). Ces résultats sont à considérer avec prudence, étant donnée la difficulté des modèles à bien restituer les étiages sur de nombreux bassins (voir notamment les critères NS(lnQ) dans le Tableau 1) et le caractère fortement influencé des débits notamment sur les grands bassins versants.

Tous les modèles projettent des étiages plus sévères sur les grands bassins versants français, avec des résultats cependant très hétérogènes (Figure 7) : le Rhône à Beaucaire pourrait subir une baisse du QMNA5 de 20 à $50 \%$; la Garonne à Lamagistère pourrait voir son QMNA5 baisser de 5 à $70 \%$ à l'horizon 2050. Cette hétérogénéité entre résultats des modèles provient en partie d'approches très différentes dans la simulation des étiages : bien que la version d'IsbaModcou utilisée ici n'ait pas de représentation détaillée des nappes, elle est cependant plus complexe que celle de GR4J qui ne repose que sur la vidange de ses deux réservoirs. Malgré cela, la qualité de simulation des étiages d'IsbaModcou en temps présent n'est pas meilleure que celle de GR4J. Ces tendances simulées par les modèles semblent quand même prolonger des tendances à la baisse qui sont déjà observables sur certains bassins, notamment dans la zone pyrénéenne et le Pays basque [Renard et al., 2006].

\section{III.5. Évolution des crues}

Compte tenu des divergences entre les projections en période de hautes eaux, on ne peut détecter de changement significatif des débits journaliers maximaux annuels de période de retour 10 ans (QJXA10) sur la majeure partie du territoire. On note en effet une grande variabilité des résultats selon les 14 projections et pour les différents bassins français, comme l'illustrent la Figure 8 et la Figure 9,

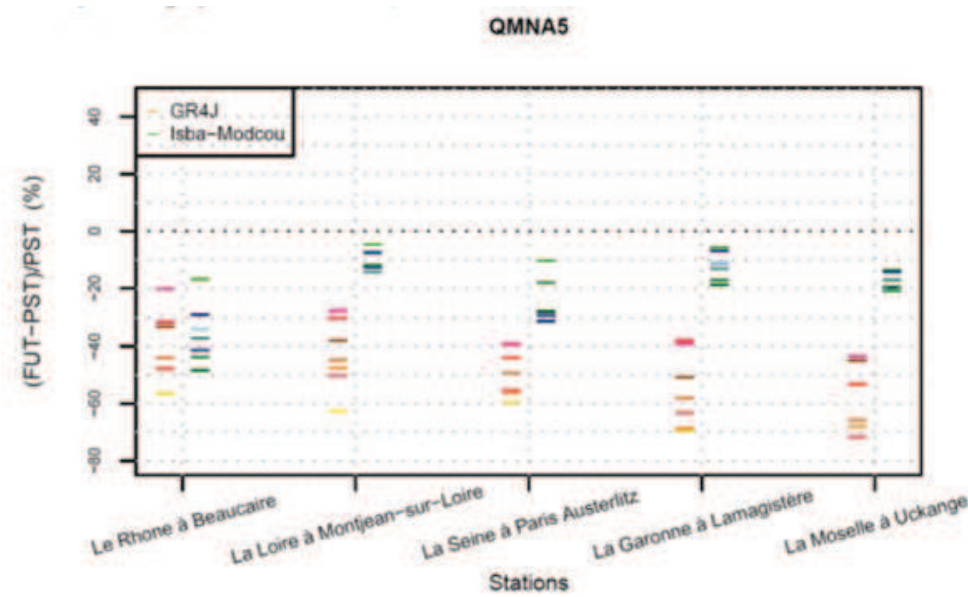

Figure 7 : Évolutions relatives possibles (en \%) du QMNA5 entre 1961-90 et 2046-65 : Résultats des 14 projections pour cinq grands bassins versants (à gauche: GR4J ; à droite: Isba-Modcou). 


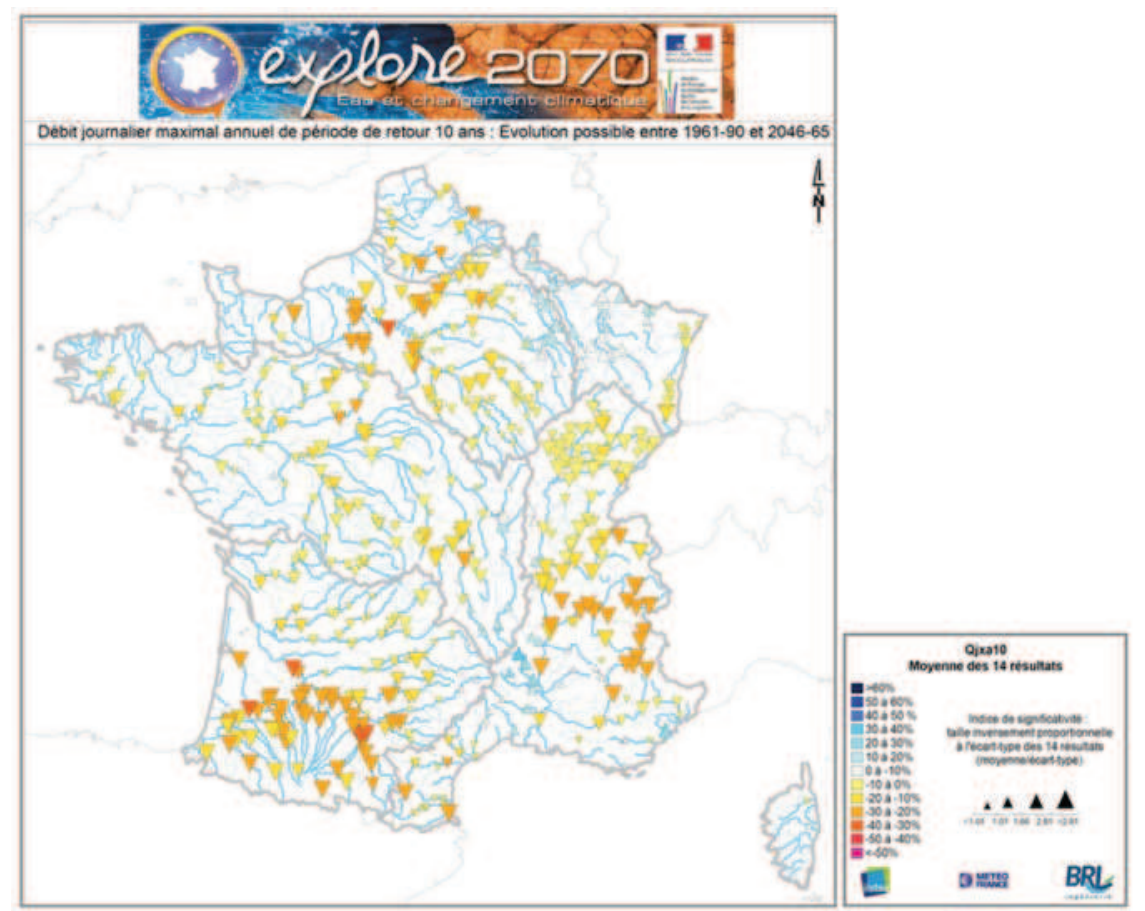

Figure 8 : Évolutions relatives possibles (en \%) du QJXA10 entre 1961-90 et 2046-65 : Résultats moyens établis sur les 14 simulations (2 modèles hydrologiques $\times 7 \mathrm{MCG})$.

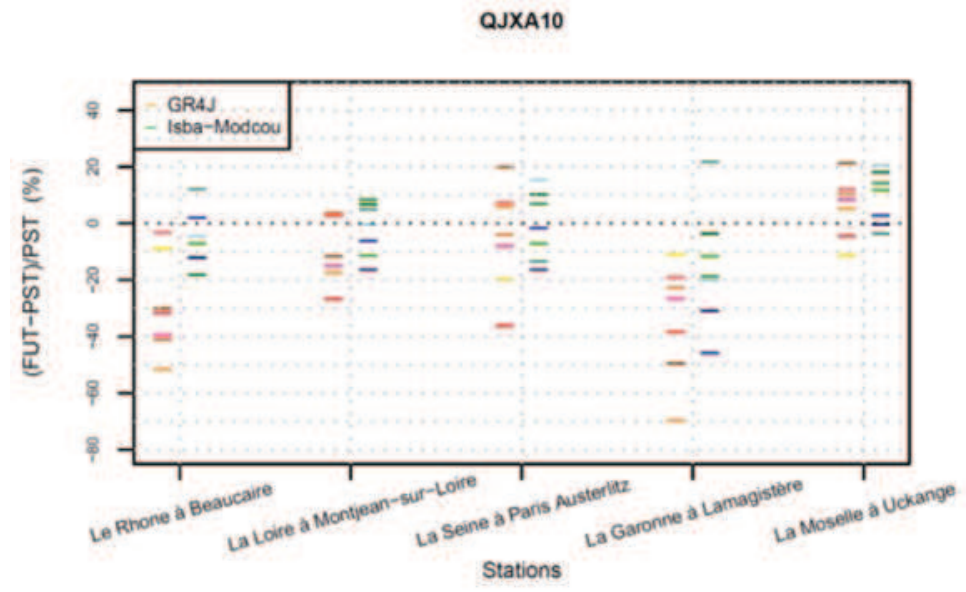

Figure 9 : Évolutions relatives possibles (en \%) du QJXA10 entre 1961-90 et 2046-65: Résultats des 14 projections pour cinq grands bassins versants (à gauche: GR4J ; à droite : Isba-Modcou).

où le ratio entre la moyenne des tendances simulées et leur écart-type est bien plus élevé que pour les étiages. Cependant, l'intensité des crues pourrait augmenter dans les Cévennes, et dans le Nord-est de la France (partie Est du district Rhin-Meuse). Les zones de haut relief (Alpes, Pyrénées, Jura), la rive gauche de la Garonne et les 2/3 ouest du district Seine-Normandie sont des zones pour lesquelles le QJXA10 pourrait baisser à l'horizon 2050 (Figure 8). Les évolutions sur les crues sont potentiellement fortement dépendantes de la méthode de descente d'échelle climatique choisie [par ex. Quintana-Seguí et al., 2011]. Une seule méthode de descente d'échelle ayant été testée ici, il convient de rester prudent sur la significativité des évolutions simulées.

\section{III.6. Incertitudes}

L'analyse des 14 résultats permet de comparer la variabilité des résultats pour les sept GCMs et pour les deux modèles hydrologiques. La Figure 10 résume les résultats issus de ces 14 chaînes de simulation. Cette analyse permet d'affirmer que :

- le poids relatif des différentes sources d'incertitudes (modèle hydrologique, GCM) dans les projections hydrologiques semble être fonction de la variable hydrologique considérée, ce qui rejoint les conclusions d'autres études [Sauquet et al., 2012] ;

— de façon générale, les résultats sont moins variables pour les moyennes annuelles que pour les extrêmes ; 

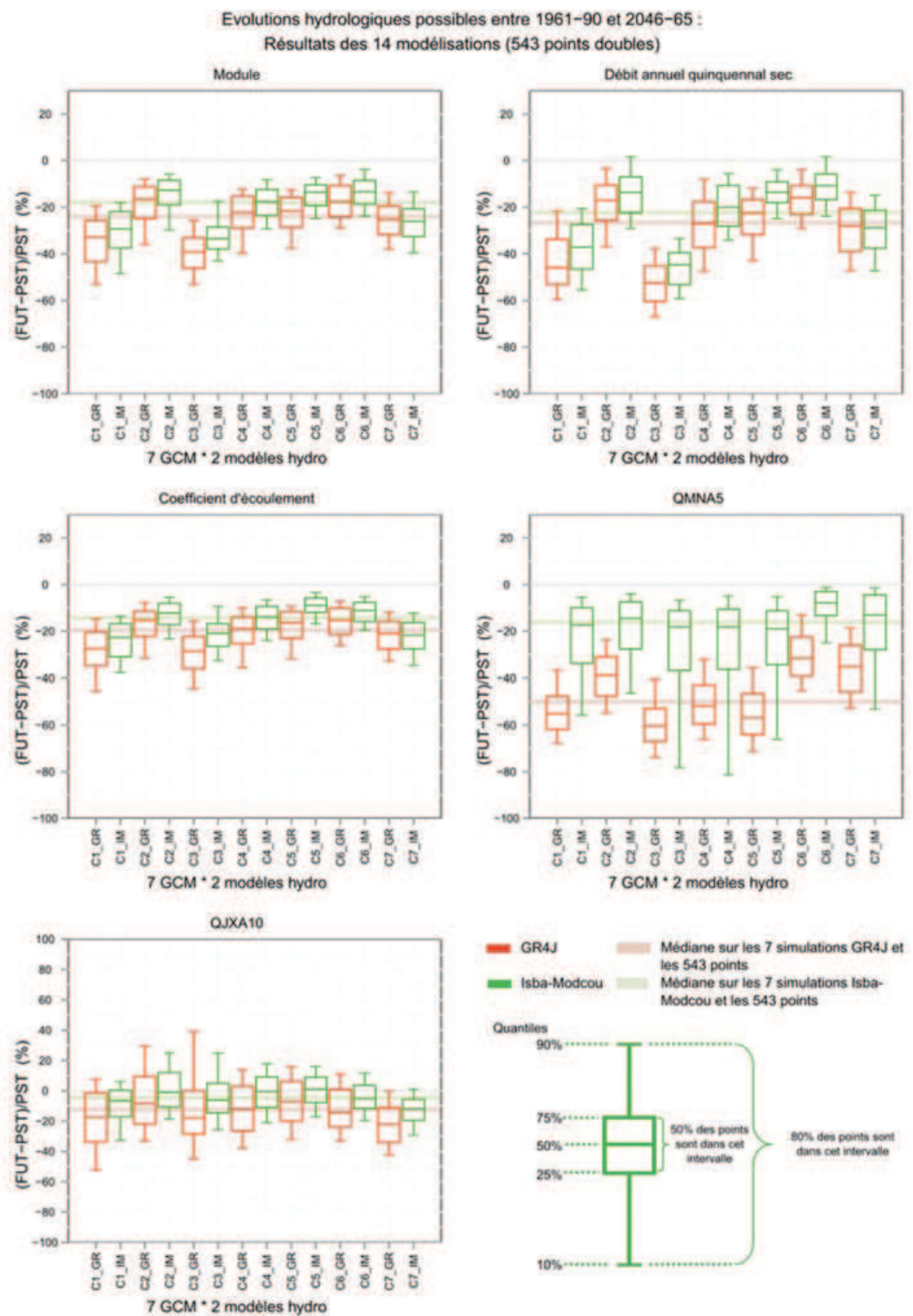

Figure 10 : Graphes en boîtes à moustache rassemblant les résultats des 14 projections (deux modèles hydrologiques GR4J et Isba-Modcou forcés par sept GCMs) au droit des 543 points de calcul communs d'Explore 2070. Chaque boîte synthétise les résultats issus d'un couple de modèle (GCM-Modèle hydrologique) sur les 543 points.

— pour le module et le débit quinquennal sec annuel, la variabilité des résultats semble venir de façon prépondérante des modèles climatiques, plutôt que des modèles hydrologiques. Même s'il existe des écarts entre ces derniers (GR4J étant souvent plus pessimiste qu'Isba-Modcou sur ces variables), ceux-ci sont globalement en accord (Figure 5 et Figure 10) ;

- pour le coefficient d'écoulement (rapport des débits et pluies moyens) et le QJXA10, on n'identifie pas de source d'incertitude prépondérante (Figure 9 et Figure 10);

- pour le QMNA5, l'écart entre les résultats vient de façon prépondérante des modèles hydrologiques (Figure 7 et Figure 10). Notons cependant que c'est en période d'étiage que la disparité des performances entre les modèles hydrologiques semble la plus forte (voir critère $\mathrm{NS}(\operatorname{lnQ})$ au Tableau 1), ce qui pourrait expliquer ce résultat.

L'utilisation de plusieurs modèles climatiques et hydrologiques est ainsi source d'une variabilité importante des changements hydrologiques calculés. Il faut cependant garder à l'esprit que certaines sources d'incertitudes n'ont pas été quantifiées. On peut citer notamment les incertitudes liées au choix du scénario d'émission de gaz à effet de serre, à la méthode de descente d'échelle, qui peut fortement impacter les résultats [Quintana Seguí et al., 2010, 2011], à la méthode d'estimation de l'évapotranspiration potentielle [Kingston et al., 2009], à la transposabilité temporelle des modèles hydrologiques et à leurs capacités d'extrapolation [Coron et al., 2012], liée à l'hypothèse d'un biais 
constant entre présent et futur. Il est enfin vraisemblable que les sources principales d'incertitudes varient selon l'indicateur hydrologique considéré [Sauquet et al., 2012]. Par exemple, le QMNA5, dont la valeur repose en grande partie sur la capacité de restitution retardée de l'eau stockée dans les nappes, et dont la prise en compte fait l'objet d'un traitement très différent entre GR4J et Isba-Modcou, est la variable qui présente la plus grande dépendance au modèle utilisé (Figure 10).

\section{DISCUSSIONS ET CONCLUSION}

Les résultats présentés ici sur la métropole montrent une diminution significative globale de la ressource à l'échelle du territoire avec, pour une majorité des cours d'eau, des étiages plus marqués. Les évolutions sur les crues, elles, sont plus hétérogènes.

Ces résultats sont en accord avec les résultats des études antérieures menées sur la France, bien que l'amplitude des changements projetés diffère sensiblement pour certaines études, en raison des choix d'hypothèses et de modélisations différents. En particulier, les baisses des débits estivaux projetées sur la Garonne sont cohérentes avec les simulations du projet Imagine2030 [Sauquet et al., 2009] et les résultats de Tisseuil et al. [2010], mais plus marquées que les changements calculés par Caballero et al. [2007] au même horizon. Cette étude avait été réalisée avec des projections plus anciennes et un scénario de gaz à effet de serre B2 moins impactant. Les changements simulés sur la Loire sont également en accord avec le projet antérieur ICC-HydroQUAL [Moatar et al., 2010], qui concluait à une baisse des débits moyens et d'étiage. En outre, les résultats d'Explore 2070 confirment les changements annoncés par les projets GICCSeine et RExHySS [Ducharne et al., 2011] sur le bassin de la Seine, à savoir une tendance nette à la diminution des débits moyens et des étiages, et des évolutions moins marquées et plus incertaines sur les crues. L'amplitude de la baisse des débits est cependant plus marquée pour Explore 2070 , ce qui s'expliquerait par une modélisation différente de l'évapotranspiration potentielle : les formulations utilisées dans les deux projets sont légèrement différentes, ce qui peut entraîner sur certains bassins des évolutions différentes de l'ETP, ce qui se répercute ensuite sur les débits [voir Chazot et al., 2012, pour une analyse plus détaillée].

En parallèle des travaux réalisés sur la métropole, des projections ont également été réalisées sur les départements d'Outre-Mer (DOM - Guadeloupe, Guyane, Martinique, Réunion). Les résultats sont affectés de fortes incertitudes, en raison du faible niveau d'information climatique disponible (peu de projections, pas de méthode de descente d'échelle fine, présence d'événements cycloniques très délicats à modéliser, etc.) et des performances modestes de la modélisation hydrologique. Compte tenu de ces incertitudes et des résultats obtenus, il a semblé délicat de dégager des changements clairs à l'échelle de chacun de ces DOM, et il est souhaitable de poursuivre ces travaux dans le cadre de projets de recherche si l'on souhaite dégager des évolutions avec plus de confiance sur ces régions.

Les incertitudes énoncées et la variabilité des résultats obtenus sont souvent d'un ordre de grandeur comparable aux changements simulés, ce qui invite à la prudence sur la significativité des valeurs absolues des tendances. La convergence des résultats sur les grandes tendances constitue cependant une base pour encourager des politiques d'adaptation.
Les résultats présentés ici ont été utilisés dans le cadre d'Explore 2070 par d'autres lots thématiques (eau souterraine, biodiversité, etc.) et ont servi à la définition de stratégies d'adaptation. Malgré les incertitudes présentes dans les résultats, celles-ci ne doivent pas empêcher d'agir dès à présent. La France doit se préparer à une situation globalement plus sèche, avec des écoulements réduits mais aussi des sols plus secs, plus tôt dans l'année, avec toutes les conséquences agricoles induites. En termes de crues, les résultats établis sont moins nets et rien ne permet d'affirmer clairement aujourd'hui qu'il faille se préparer, à temps de retour équivalent, à des évènements plus intenses à l'échelle nationale, quelques régions présentant cependant des évolutions plutôt à la hausse.

Cette étude à l'échelle nationale pourrait servir de base vis-à-vis d'exercices plus locaux, qui permettraient une meilleure prise en compte des données disponibles (débits naturalisés, fonctionnement des ouvrages hydraulique, etc.) et des contextes particuliers des territoires en termes de gestion, d'offre et de demande en eau. Une meilleure évaluation des changements pourrait également être obtenue en utilisant les prochaines projections climatiques produites par CMIP5 (Coupled Model Intercomparison Project Phase 5) dont les données régionalisées seront livrées en 2014.

\section{REMERCIEMENTS}

Ces travaux ont été réalisés dans le cadre du Lot Hydrologie de surface du projet Explore 2070 (http://www. developpement-durable.gouv.fr/hydrologie-de-surface. html) initié et financé par la Direction de l'Eau et de la Biodiversité du Ministère de l'écologie, du développement durable et de l'énergie. Ces travaux ont pu être menés à bien grâce à la mise à disposition de larges bases de données d'un certain nombre d'institutions qui sont remerciées ici (Météo-France, CERFACS, SCHAPI, Seine Grands Lacs) et grâce à l'exploitation des résultats de projets antérieurs (Imagine 2030 notamment).

\section{REFERENCES}

BoÉ J. (2007) - Changement global et cycle hydrologique : Une étude de régionalisation sur la France. Thèse de Doctorat, Cerfacs (Toulouse), Université de Toulouse III Paul Sabatier. $255 \mathrm{p}$.

Boé J., Terray L., Habets F., Martin E. (2007) - Statistical and dynamical downscaling of the Seine basin climate for hydrometeorological studies. International Journal of Climatology. 27(12) : 1643-1655

Boé J., Terray L., Martin E., Habets F. (2009) - Projected changes in components of the hydrological cycle in French river basins during the 21 st century. Water Resources Research. 45: W08426

Caballero Y., Voirin-Morel S., Habets F., Noilhan J., Le Moigne P., Lehenaff A., Boone A. (2007) - Hydrological sensitivity of the Adour-Garonne river basin to climate change. doi10.1029/2005WR004192. Water Resources Research. 43 : W07448, 19

Chazot S., Norotte T. David J. (2012) - EXPLORE 2070 - Lot Hydrologie de surface. B4 - Thermie. Rapport final du projet Explore 2070, BRLi - Irstea - Météo France, Ministère de l'Ecologie, Paris. 77 p.

Chazot S., Perrin C., Vidal J.P., Sauquet E., Chauveau M., Rouchy N. (2012) - EXPLORE 2070 - Lot Hydrologie de 
surface. A1 - Rapport de synthèse. Rapport final du projet Explore 2070, BRLi - Irstea - Météo France, Ministère de l'Ecologie, Paris. $146 \mathrm{p}$.

Chazot S., Perrin C., Vidal J.P., Sauquet E., Chauveau M., RouchY N. (2012) - EXPLORE 2070 - Lot Hydrologie de surface. B3 - Rapport détaillé DOM. Rapport final du projet Explore 2070, BRLi - Irstea - Météo France, Ministère de l'Ecologie, Paris. 158 p.

Coron L., V. Andréassian, C. Perrin, J. Lerat, J. Vaze, M. BouRqui, F. HENDRICKX (2012) - Crash testing hydrological models in contrasted climate conditions: an experiment on 216 Australian catchments. Water Resources Research. 48(5) : W05552

Ducharne A, Sauquet E, Habets F, Deque M, Gascoin S, Hachour a, Martin E, Oudin L, Page C, Terray L, Thiery D, VIENNOT P (2011) - Evolution potentielle du régime des crues de la Seine sous changement climatique. La Houille Blanche, $n^{\circ} \mathbf{1}: 51-57$

Habets F., A. Boone J. L. Champeaux P. Etchevers L. Franchisteguy E. Leblois E. Ledoux P. Le Moigne E. Martin S. Morel J. Noilhan P. Quintana Segui F. RoussetRegimbeau P. Viennot (2008) - The SAFRAN-ISBAMODCOU hydrometeorological model applied over France, doi10.1029/2007JD008548. J. Geophys. Res. 113 : D06113

Kingston D. G., M. C. Todd R. G. TAylor J. R. Thompson N. W. ARNELl (2009) - Uncertainty in the estimation of potential evapotranspiration under climate change. Geophysical Research Letters. 36 : L 20403

KLEMEŠ V. (1986) - Operational testing of hydrological simulation models. Hydrological Sciences Journal. 31(1) : 13-24

Le Moine N., V. Andreassian C. Perrin C. Michel (2007) - How can rainfall-runoff models handle intercatchment groundwater flows? Theoretical study based on 1040 French catchments. Water Resources Research. 43(6) : W 06428

Moatar F., Ducharne A., Thiéry D., Bustillo V., Sauquet E., VIDAL J.P. (2010) — La Loire à l'épreuve du changement climatique. Géosciences. 12 : 78-87

Najac J., Kitova N., Vidal J.-P., Soubeyroux J.-M., Martin E. (2010) - Caractérisation des sécheresses en France au cours du XXIeme siècle (Drought characterization in France over the $21^{\text {st }}$ century). V. Dubreuil, O. Planchon, H. Quenol et V. Bonnardot (Editors), XXIIIe Colloque de l'Association Internationale de Climatologie - Risques et changement climatique. Association Internationale de Climatologie, Rennes. 433-438

NASH J. E., J. V. Sutcliffe (1970) - River flow forecasting through conceptual models. Part I - A discussion of principles. Journal of Hydrology. 10(3) : 282-290

Oudin L., Andréassian V. Perrin C. Michel C., Le Moine N. (2008) - Spatial proximity, physical similarity and ungaged catchments: confrontation on 913 French catchments. Water Resources Research. 44 : W03413

Pagé C., Terray L., Boé J. (2008) - Projections climatiques à échelle fine sur la France pour le $21^{\text {ème }}$ siècle: les scénarii SCRATCHO8; CERFACS. $21 \mathrm{p}$.

Perrin C., Michel C., Andréassian V. (2003) - Improvement of a parsimonious model for streamflow simulation. Journal of Hydrology. 279 : 275-289

Perrin C. Vidal J.P., Chazot S. Norotte T. (2010) - EXPLORE 2070. Synthèse bibliographique - Lot Hydrologie de surface.
Rapport intermédiaire du projet Explore 2070. Ministère de l'Ecologie, Paris. 67 p.

Quintana-Seguí P., Habets F., Martin E. (2011) - Comparison of past and future Mediterranean high and low extremes of precipitation and river flow projected using different statistical downscaling methods. Natural Hazards and Earth System Sciences. 11 : 1411-1432

Quintana-Segui P., P. Le Moigne, Y. Durand, E. Martin, F. Habets, M. Baillon, C. Canellas, L. Franchisteguy, S. Morel (2008) - Analysis of near-surface atmospheric variables: Validation of the SAFRAN analysis over France. Journal of Applied Meteorology and Climatology. 47(1) : 92-107

Quintana-Seguí P., Ribes A., Martin E., Habets F., BoÉ J. (2010) - Comparison of three downscaling methods in simulating the impact of climate change on the hydrology of Mediterranean basins. Journal of Hydrology. 383 : 111-124

Renard B., M. Lang, P. Bois, A. Dupeyrat, O. Mestre, H. Niel, J. Gailhard, C. Laurent, L. Neppel Et E. Sauquet (2006) Evolution des extrêmes hydrométriques en France à partir de données observées. La Houille Blanche. 6 : 48-54

Sauquet E., Dupeyrat A., Hendrickx F., Labedade R., Samie R., Vidal, J-P., Perrin C., Boudhraa H., Gaëckler M. (2009) - Impacts anthropiques et étiages de la Garonne à l'horizon 2030. Actes du "Colloque 193 SHF : Etiages, Sécheresses, Canicules rares et leurs impacts sur les usages de l'eau", Lyon, France, 7-8/10/ 2009.

Sauquet E., Vidal J.-P., Perrin C., Bourgin P.-Y., Chauveau M., Снаzот S., Rouchy N. (2012) - Climate and hydrological uncertainties in projections of flood and low-flows in France. Geophysical Research Abstracts. 14 : 5424

Soubeyroux J.-M., Martin E., Franchisteguy L., Habets F., Noilhan J., Baillon M., Regimbeau F., Vidal J.-P., Le Moigne P., Morel S. (2008) - Safran-Isba-Modcou (SIM) -- Un outil pour le suivi hydrométéorologique opérationnel et les études. La Météorologie. 63 : 40-45

Soubeyroux J.-M., Vidal J.-P., Baillon M., Blanchard M., Ceron J.-P., Franchisteguy L., Regimbeau F., Martin E., VINCENDON J.-C. (2010) - Caractérisation et prévision des sécheresses et étiages en France à partir de la chaîne hydrométéorologique Safran-Isba-Modcou. La Houille Blanche. 5 : 30-39

Soubeyroux J.-M., Vidal J.-P., Najac J., Kitova N., Blanchard M., Dandin P., Martin E., Page C., Habets F. (2011) - Projet ClimSec - impact du changement climatique en France sur la sécheresse et l'eau du sol (ClimSec project - impact of climate change on drought and soil moisture in France, in French). Rapport final, Météo-France, CNRM/GAME, Cemagref, CERFACS and UMR Sisyphe.

Tisseuil C., VRac M., Lek S., Wade A.J. (2010) - Statistical downscaling of river flows. Journal of Hydrology. 385(1-4) : 279-291

Vidal J.P., Martin E., Kitova N., Soubeyroux J.-M. (2012) Evolution of spatio-temporal drought characteristics: validation, projections and effect of adaptation scenarios. Hydrology and Earth System Sciences. 16 : 2935-2955

Vidal J.-P., Martin E., Franchisteguy L., Baillon M., Soubeyroux J.-M. (2010) - A 50-year high-resolution atmospheric reanalysis over France with the Safran system. International Journal of Climatology. 30(11) : 1627-1644 